\title{
Philosophiques
}

\section{Être vrai}

\section{Markus Gabriel}

Volume 45, numéro 1, printemps 2018

URI : https://id.erudit.org/iderudit/1048624ar

DOI : https://doi.org/10.7202/1048624ar

Aller au sommaire du numéro

Éditeur(s)

Société de philosophie du Québec

ISSN

0316-2923 (imprimé)

1492-1391 (numérique)

Découvrir la revue

Citer ce document

Gabriel, M. (2018). Être vrai. Philosophiques, 45(1), 239-247.

https://doi.org/10.7202/1048624ar

Ce document est protégé par la loi sur le droit d'auteur. L'utilisation des services d'Érudit (y compris la reproduction) est assujettie à sa politique d'utilisation que vous pouvez consulter en ligne.

https://apropos.erudit.org/fr/usagers/politique-dutilisation/
Cet article est diffusé et préservé par Érudit.

Érudit est un consortium interuniversitaire sans but lucratif composé de l’Université de Montréal, l'Université Laval et l'Université du Québec à Montréal. Il a pour mission la promotion et la valorisation de la recherche. https://www.erudit.org/fr/ 


\section{Être vrai}

\section{MARKUS GABRIEL}

Institut für Philosophie, Universität Bonn

Faisant suite aux livres Le bruit du sensible et Éléments de philosophie réaliste, L'adresse du réel est la contribution la plus récente de Jocelyn Benoist au débat du $\mathrm{XXI}^{\mathrm{e}}$ siècle portant sur le réalisme. C'est un honneur pour moi de constater que Benoist a profité de cette occasion pour consacrer quelquesunes de ses profondes réflexions épistémologiques et sémantiques à des problèmes qui ont constitué le cœur de nos conversations des dernières années, portant sur la question de savoir quelles sont les formes de réalisme les plus appropriées.

À mon avis, Benoist est actuellement le philosophe français, dont les travaux portent sur les principaux domaines de la philosophie théorique, le plus intéressant et le plus original. Ce qui m'impressionne le plus dans son travail, c'est l'honnêteté intellectuelle avec laquelle il énonce les détails de son cheminement qui le mène à surmonter les impasses du réalisme métaphysique, c'est-à-dire de tout cadre dans lequel la philosophie est essentiellement pensée comme une enquête portant sur les objets qui meubleraient une réalité purement indépendante de l'esprit. L'approche radicalement contextualiste de Benoist se défait effectivement de l'idée erronée selon laquelle le réalisme aurait d'abord affaire avec ce qui est extramental. Il nous rappelle en ce sens que le réalisme devrait être en mesure de rendre compte du fait qu'en tant que sujets qui connaissons et pensons, nous sommes présents dans la réalité que nous percevons et théorisons. La réalité n'est pas une masse de matière anonyme qui affecte notre sensibilité.

Je vais profiter de l'occasion qui m'est accordée pour examiner certains des différends que nous pourrions avoir. Avant de souligner un potentiel point central de divergence, je souhaite cependant réaffirmer que nous avons beaucoup plus d'opinions en partage qu'il n'y en a qui nous séparent. En fait, j'ai développé ma propre position grâce à la coopération étroite que j'ai entretenue avec Benoist au cours des deux dernières années à Paris et à Bonn, laquelle a eu de profondes répercussions sur mes positions concernant les problèmes qui entourent le non-existant. Même si, dans L'adresse du réel, Jocelyn Benoist traite explicitement aussi de cet aspect de notre dialogue, je vais pour ma part ici me concentrer sur l'aspect de la relation entre l'existence et l'être-vrai qui semble être à l'arrière-plan de certaines des inquiétudes que mon confrère peut entretenir à l'égard de mon pluralisme ontologique et sa compatibilité avec ses vues contextualistes. 
Frege est reconnu pour avoir soutenu que la logique découvre «les lois de l'être vrai ${ }^{1}{ }$. Cela passe parfois inaperçu en raison du fait que la phrase allemande "Gesetze des Wahrseins" a en anglais inadéquatement été traduite par «laws of truth ${ }^{2}$ ». Pourtant, comme il appert clairement des premières lignes du chapitre "La pensée» et de tous les autres passages dans lesquels Frege s'oppose au psychologisme, c'est grâce au départage entre l'être vrai (Wahrsein) et le tenir pour vrai (Fürwahrhalten) que Frege précise ce qu'est la logique. L'expression «Fürwahrhalten» a elle aussi été erronément traduite par le terme "assertion» (assertion) dès l'ouverture de la traduction anglaise classique de "The Thought ${ }^{3}$ ", et ce, alors que Frege distinguera l'acte de juger (tenir pour vrai) et l'assertion plus tard dans son essai.

Ces erreurs de traduction dans les passages introductifs de l'essai de Frege expliquent en partie l'opinion erronée, mais aujourd'hui répandue, selon laquelle Frege aurait cru que la vérité, et conséquemment le sens, est normative, c'est-à-dire de l'ordre de l'exigence.

Dans sa discussion de mon usage de Frege au sein de mon ontologie des champs de sens, Benoist se sert de l'idée de la normativité du sens et de la vérité afin de mettre le doigt sur la source du désaccord qui sépare à quelques endroits nos conceptions du réalisme. Nous sommes clairement tous les deux d'accord quant au fait qu'il faut s'opposer à la sorte de réalisme métaphysique qui est caractéristique des supposées métaphysiques analytiques. Aucun de nous deux ne croit qu'il fait sens de chercher à obtenir une description de la nature substantielle de la réalité à partir du point de vue de la philosophie (ni par ailleurs d'aucune autre discipline). Il n'y a pas de réalité «là-bas au-dehors» ( «out there») dont l'architecture puisse être rendue explicite par une théorisation métaphysique. Pourtant, nous avons dans une certaine mesure plusieurs raisons de répudier un tel projet, raisons qui expliquent que nous sommes tous les deux en faveur d'une position réaliste, et qui nous distinguent de la réaction excessive de l'antiréalisme à l'échec du réalisme métaphysique (comme chez Dummett, Rorty, Putnam, Derrida).

Benoist maintient que le réalisme métaphysique se méprend sur la contextualité de la pensée et du langage, là où je pense que la contextualité en question fait elle-même partie de la réalité. En fait, comme Benoist l'observe avec raison ${ }^{4}$, j'abandonne complètement l'idée selon laquelle le réalisme aurait quoi que ce soit à voir avec l'idée d'une réalité indépendante de l'esprit. L'esprit (quoi qu'il soit par ailleurs) est aussi réel que n'importe quel

1. Gottlob Frege, Écrits logiques et philosophiques, trad. C. Imbert, Paris, Éditions du Seuil, I97I, p. I70.

2. Gottlob Frege, The Thought: A Logical Inquiry, trad. M. P. Geach, Mind. A Quarterly Review of Psychology and Philosophy, vol. 65, n' 259, juillet I956, p. 289.

3. Frege, The Thought: A Logical Inquiry, p. 290.

4. Jocelyn Benoist, L'adresse du réel, Paris, Librairie philosophique J. Vrin, 20I7, p. 23. 
autre objet. Par conséquent, il faudrait déjà pour commencer ne pas formuler la question du réalisme sous la forme d'une relation entre ce qu'il y a et comment nous le pensons.

C'est dans ce contexte que j'ai défendu une conception que j'ai nommée «descriptivisme ontologique ${ }^{5}$ ». Le descriptivisme ontologique associe les objets (que l'on ne doit pas confondre avec les noms propres!) aux groupes de sens frégéens, ou du moins d'après ce que je comprends du "sens frégéen ». Selon ma lecture, Frege serait d'avis, tout comme moi, que le sens est une propriété des choses en elles-mêmes. Benoist serait certainement d'accord avec l'idée selon laquelle le sens ne peut, en général, pas venir à l'existence à titre d'effet secondaire de l'expression de la pensée humaine. Les sens frégéens sont découverts étant donné que les pensées frégéennes sont.

À ce propos, Frege ne laisse planer aucun doute quant au fait qu'il pense les lois de l'être vrai par analogie aux lois de la nature.

Quant aux lois de la nature, elles constituent l'élément général des événements naturels auxquels ceux-ci ne manquent jamais de se conformer. C'est plutôt en ce sens que je parle de lois de l'être vrai. Encore ne s'agit-il pas dans ce cas d'un événement mais d'un être'.

C'est ici qu'intervient Jocelyn Benoist. Il se demande alors comment une pensée fausse est possible si le sens est conçu comme une propriété des objets. Si, en général, les lois de la logique décrivent ce qui découle de quelque chose, comment pourrait-il alors y avoir une pensée fausse? Plus spécifiquement, Benoist demande: "Si le sens est propriété de la chosemême, comment une description pourrait-elle jamais être incorrecte ?7 " Je répondrai à cette question en disant qu'il nous faut distinguer deux choses. À un certain niveau, il est facile de dire comment une description peut être incorrecte. Une description au sens strictement logique est incorrecte dans le contexte (Zusammenhang) d'une phrase qui exprime une vérité ou une fausseté. Si une phrase exprime une fausseté, la pensée qu'elle exprime est fausse. Pour expliquer cela, nous pourrions invoquer la part descriptive de la pensée, c'est-à-dire le prédicat logique ou le concept selon le vocabulaire employé par Frege.

L'objet d'une pensée de premier niveau n'est pas correct ou incorrect. Il est tout simplement et, en ce sens, il appartient bel et bien à quelque chose de similaire à la catégorie du factuel chez Benoist. Toutefois, il est également vrai qu'en un certain sens précis, les objets frégéens ne sont pas factuels. Les faits sont des pensées vraies, alors que les objets ne sont pas des pensées, mais font partie des pensées. Les objets sont ce à propos de quoi peuvent

5. Markus Gabriel, Sinn und Existenz: eine realistische Ontologie, Berlin, Suhrkamp, 20I6, p. 38 et suiv.; p. 87 ; p. 224 et suiv.

6. Frege, Écrits logiques et philosophiques, p. I70.

7. Benoist, L'adresse du réel, p. 98. 
être les pensées. Dans Fields of Sense, j'ai défendu l'idée selon laquelle il n'y a pas d'objets s'il n'y a pas de pensées frégéennes. Cette idée équivaut essentiellement à l'idée qu'il n'y a pas d'objets s'il n'y a pas quelque chose qui est vrai à propos d'eux. Les objets font essentiellement partie des faits. Le factuel n'est pas seulement fait d'objets. C'est pourquoi je rejette les ontologies plates, comme le remarque avec raison Benoist ${ }^{8}$.

Mais qu'en est-il de nos pensées? Comment peuvent-elles être incorrectes si le sens fait partie de la réalité ? Je considère que la réponse à cette question est simple elle aussi: il est possible de juger que p même si $\neg$ p, et vice versa. Frege le dit de la façon suivante: «L'erreur et le préjugé ont leur cause, tout comme la connaissance juste. L'opinion fausse et l'opinion vraie adviennent l'une comme l'autre selon des lois psychologiques9." Benoist est très conscient du fait que je suis en désaccord avec Frege sur ce point. Je ne crois pas que l'erreur et la connaissance sont causées dans le domaine de la psychologie. C'est le psychologisme résiduel de Frege. Frege reste sujet au psychologisme lorsqu'il est question de l'acte de penser, même s'il rejette par ailleurs le psychologisme dans le cas des pensées ${ }^{10}$.

À l'encontre de ce que je propose, Benoist veut maintenir l'idée que les pensées fausses peuvent survenir dans la vie mentale de l'homme en raison d'une normativité qui, pour une raison ou pour une autre, ne serait pas couverte par une réponse à la question Qu'y a-t-il? D'après lui, la pensée au sens d' "acte de penser» n'appartiendrait pas à la réalité:

Ma divergence avec Markus Gabriel trouve probablement son fondement dans le motif, essentiel pour moi, de la différence catégoriale entre le normatif et le factuel. La réalité, pour moi, revêt fondamentalement l'aspect de la factualité au sens où, par définition, elle n'est jamais que ce qu'elle est. Une réalité n'est pas vraie ou fausse; elle est. En revanche, ce qu'il faut retenir de ce que Frege appelle «sens", c'est précisément sa capacité à être vrai ou faux, ou disons plus largement correct ou incorrect. Le sens, chez Frege, est fondamentalement une instance normative. Par là même, il ne peut pas être une propriété des choses, mais un format de détermination de ces mêmes choses, suivant lequel ces propriétés sont correctement ou incorrectement saisies ${ }^{11}$.

Contrairement à ce que conclut Jocelyn Benoist, j'aimerais établir une distinction entre «saisir l'incorrect» et «incorrectement saisir». Les passages de Frege auxquels j'adhère me portent à croire qu'il n'y a rien de tel qu'un «incorrectement saisir ». L'erreur consiste à saisir quelque chose d'incorrect et non pas à saisir incorrectement. Lorsque nous saisissons quelque chose d'incorrect, nous ne saisissons pas ce que nous voulions saisir, mais

8. Benoist, L'adresse du réel, p. 8.

9. Frege, Écrits logiques et philosophiques, p. I7I.

10. Markus Gabriel, Fields of Sense: A New Realist Ontology, Edinburgh, Edinburgh University Press, 20I 5, p. 338-358.

11. Benoist, L'adresse du réel, p. 98. 
plutôt quelque chose d'autre. Si je confonds Jules avec Jim, je voulais porter un jugement véridique sur Jim, mais je porte à la place un jugement faux sur Jules. C'est d'ailleurs pourquoi je peux apprendre quelque chose de mes erreurs, par exemple, que Jim est étonnamment semblable à Jules.

À cet égard, un autre enjeu crucial dépend de la signification que nous attachons au terme "réalité ». Frege situe clairement la vérité et la fausseté dans l'être. Les pensées peuvent être vraies ou fausses. Il n'y a pas de pensée qui puisse, selon Frege, osciller entre les deux. Chaque pensée frégéenne est ou bien vraie, ou bien fausse. Si nous nous fions au critère de Benoist, les pensées frégéennes semblent appartenir à la réalité car elles sont. Selon ma lecture de Frege, il n'y a tout simplement pas de "capacité à être vrai ou $f^{\prime} x^{12}$ »; il n'y a qu'un ensemble de faits établissant qu'une pensée est en réalité vraie ou fausse.

Il n'y a pas de niveau intermédiaire. L'illusion d'un tel niveau intermédiaire résulte de contextes dans lesquels nous considérons une pensée en excluant le fait qu'elle est en fait vraie ou fausse, par exemple dans le discours indirect, dans les questions, dans la recherche, et ainsi de suite. Frege traite de ces phénomènes de façon à ne justement jamais compromettre l'orientation réaliste de sa position suivant laquelle nous découvrons des pensées (des entités qui sont objectivement vraies ou objectivement fausses): jamais nous ne les créons ${ }^{13}$. D'après moi, Jocelyn a seulement partiellement raison lorsqu'il affirme:

En objectivant l'univers kantien des représentations et en faisant des «sens» (Sinne), Frege a encore maintenu l'idée d'une instance intermédiaire entre le monde et nous. Au contraire, selon la conception défendue par Markus Gabriel, nos significations nous engagent directement vis-à-vis du monde, parce qu'elles ont purement et simplement une portée ontologique. De fait, nos significations ouvrent autant de dimensions du monde ${ }^{14}$.

L'erreur de Frege réside dans son concept de représentation (Vorstellung). L'interface qui se situe entre nous et la réalité n'est pas selon Frege de l'ordre du sens. Le sens est une propriété de la façon d'être des choses, et non pas une propriété de nos représentations de la réalité. Toutefois, Frege fait de la place pour un domaine intermédiaire qui est mal conçu puisqu'il n'est pas capable de résoudre la question de savoir comment, en tant que penseurs finis, nous pourrions un jour saisir le sens si nous ne pouvons juger qu'au moyen de représentations.

Il est possible de concéder du terrain à l'inquiétude de Benoist à l'égard de ma reconstruction ontologique austère et réaliste du cadre frégéen. Les objets frégéens semblent en effet être hors du domaine du sens, pour ainsi

12. Benoist, L'adresse du réel, p. 98.

13. Frege, Écrits logiques et philosophiques, p. I9I.

14. Benoist, L'adresse du réel, p. 69. 
dire. Dans toute analyse des composantes d'une pensée de premier niveau (d'une pensée qui ne porte pas sur une pensée), il y aura une place pour un objet, là où un objet n'est pas lui-même un concept. Sans entrer dans le mécanisme qui permet à Frege lui-même d'affirmer une telle chose, il est correct de dire que certaines pensées ont trait à quelque chose qui n'a pas lui-même dans la pensée la forme logique d'une description.

Disons que je juge que la bouteille qui se trouve là-bas est vide. La pensée que je saisis ainsi - que la bouteille est vide — dépend de la bouteille. Elle a trait à la bouteille. Ce qu'elle me dit à propos de la bouteille, c'est qu'elle est vide. Le concept d'être-vide est tel que plusieurs choses peuvent être vides. Pourtant, dans la pensée qu'elle est vide, il n'y a rien concernant cette bouteille qui me renvoie à d'autres objets. Cela ne doit pas nous induire à croire qu'il y aurait une réalité de premier niveau qui serait composée de tous les objets. Il n'y a pour ainsi dire rien qui puisse être un objet en lui-même, c'est-à-dire indépendamment d'une pensée qui traite de la façon dont est une chose.

Or nous ne sommes pas face à une forme d'idéalisme ontique qui prétendrait que seuls existent les contenus des actes de penser réalisés par un être pensant (que ce soit Dieu, les anges, les humains, les ordinateurs, les animaux, etc.). Afin d'éviter tout malentendu de ce type, je parle de champs de sens au lieu de pensées, même si la construction théorique de l'ontologie des champs de sens est inspirée par ce que Frege entend par "pensée».

Les objets sont donc ce qu'ils sont une fois qu'un champ de sens est mis en place. Un objet a ses propriétés dans un champ de sens. Pourtant, cela ne veut pas dire qu'un objet diffère de toutes les vérités (les faits) qui l'impliquent. Au contraire, un objet est identique à tout ce qui est vrai de lui. Cette bouteille est, notamment, une bouteille. C'est la raison pour laquelle je peux juger que ceci est une bouteille. La bouteille n'est pas une réalité impénétrable simplement indexée. Elle a des propriétés, dont celle d'être une bouteille. Selon ma conception, toute bouteille réelle peut également avoir la propriété d'avoir été mal identifiée par quelqu'un. Si quelqu'un a fait une erreur à propos de cette bouteille, il a saisi une pensée fausse. Cette bouteille apparaît tout autant dans les pensées vraies que dans les pensées fausses. Une pensée fausse n'est jamais entièrement détachée de l'objet qu'elle vise. Sans quoi, elle ne serait même pas fausse.

À cet égard, je ne suis pas encore sûr d'être d'accord avec l'idée de Benoist selon laquelle un objet "n'est rien d'autre qu'une norme d'identification $^{15}$ ». S'il veut dire que je peux porter des jugements vrais ou faux à propos de n'importe quel objet, je pourrais accepter une version étroite de ce qu'il affirme. Je pense à ceci : il y a des objets à propos desquels personne n'aura jamais une pensée de re vraie ou fausse. Il est vrai que je peux penser à ces objets de dicto, comme je viens de le faire. Cependant, tous les objets

15. Benoist, L'adresse du réel, p. 23. 
que nous nous trouvons à connaître sont tels qu'ils prescrivent quelles pensées à leur propos sont vraies et lesquelles sont fausses. Ils sont des normes dans la mesure où leur existence même distingue les jugements qui sont corrects et ceux qui sont incorrects. Un jugement à propos d'un objet doit être dit "correct » s'il saisit la vérité, et "incorrect» dans le cas contraire. Le fait que l'objet lui-même décide de quel côté se situe un jugement (respectivement : correct ou incorrect) peut alors être caractérisé en termes de «norme d'identification".

Je ne suis pour l'instant pas certain de comprendre comment Benoist souhaite manier la distinction qu'il opère entre la structure et la norme ${ }^{16}$. S'il situe les objets du côté de la norme plutôt que du côté des structures, il semble être attaché à l'idée selon laquelle n'eût été les normes, il n’y aurait aucun objet. Si une norme requiert que quelqu'un soit soumis à elle, et si les objets sont des normes, alors nos pratiques entourant la détermination des normes par l'instauration d'un contexte seraient à l'origine (causale?) des objets.

Certes, il y a lieu de parler de l'idée d'une norme d'identification. Mais qu'en est-il alors de la composition des structures? Je place tant les objets que leurs sens au niveau des structures. Et je me permets tout de suite d'ajouter que les actes de penser, les jugements, font tout aussi partie du réel que n'importe quelle autre structure. Ma pensée qu'il pleut fait partie de la même réalité que le fait qu'il pleut. Si je juge qu'il pleut (en raison du fait que je vois un ciel gris et des gouttes de pluie sur ma fenêtre, par exemple) alors qu'il ne pleut pas (ma fenêtre a urgemment besoin d'être lavée), le fait que j'aie commis une erreur fait partie de la réalité. Compte tenu de ces considérations, je suis en désaccord avec le dualisme normatif entre le réel et notre référence à celui-ci proposé par Benoist:

Le réel en lui-même n'a pas besoin du sens pour avoir sa propre plénitude, sa propre densité d'être: il est de toute façon exactement ce qu'il est. En revanche, nous avons besoin du sens pour nous retrouver dans cette densité et c'est au moyen du sens que, contextuellement, nous arrivons à la capturer : à la thématiser et d'en faire quelque chose ${ }^{17}$.

Benoist me demande constamment d'établir une distinction entre l'existence et la réalité. Je me permets de profiter de cette occasion pour introduire une catégorie de réalité qui pourrait conceptuellement faire l'affaire. Le réel est ce à propos de quoi nous pouvons avoir raison ou tort. $\mathrm{La}$

16. «Précisons-le: il ne s'agit pas de dire que le réel est fait de structures plutôt que de normes, comme si, là encore, une chose s'opposait à l'autre et nous écartait de l'autre. Les normes, telles que je les conçois — c'est là mon réalisme — n'ont d'autre vocation que d'appréhender les structures et réciproquement la structure est exactement ce qui est appréhendé par la norme, là où celle-ci est appliquée de la façon qu'il faut dans le contexte qu'il faut» (Benoist, L'adresse du réel, p. 105-106).

17. Benoist, L'adresse du réel, p. I05. 
réalité est une caractéristique de nos jugements (nos pensées) relative au fait qu'ils peuvent être à propos du réel, c'est-à-dire qu'ils sont soumis au "contraste de l'objectivité ${ }^{18}$ ». La réalité est une catégorie de jugements. Le jugement voit le jour en même temps que l'acte de juger. Il n'en va cependant pas ainsi pour le réel. Le réel était là avant que nous n'entrions en scène. Cela ne veut pas dire que les jugements sont irréels. En jugeant, nous changeons le réel en y ajoutant quelque chose qui n'était pas là auparavant.

Pour revenir à mes réflexions initiales, j'aimerais ajouter que je suis en accord avec Frege lorsqu'il affirme qu'il y a des lois logiques ontologiques. Appelons «réel préthéorique » la façon dont les choses étaient avant que quelqu'un n'existe pour les remarquer. Le réel préthéorique est soumis à une évaluation qui tient compte des lois de la logique justement parce qu'il ne fait aucun sens de caractériser sous forme d'écart la différence entre le réel, d'une part, et la façon dont nous le traitons, d'autre part. Il n'y a pas d'écart qui séparerait en premier lieu l'esprit et le monde. Le réel n'est pas coupé en deux: ce qu'il y a et ce que nous en faisons ou pensons. Toutefois, il ne forme pas non plus une unité, un domaine globalement homogène.

Le réel n'est ni une, ni deux, mais une multitude irréductible de structures. Ces structures sont des champs de sens, dont certains sont découverts, alors que d'autres sont produits. Les lois de la logique ne font pas partie de ce que nous produisons. Nous les trouvons. Sans elles, rien n'aurait été le cas, puisqu'elles décrivent la forme de ce qui est le cas, les lois de l'être vrai. Il faut remarquer que selon ma reconstruction, les lois de l'être vrai sont ontologiques. Elles décrivent des conditions formelles qui doivent être respectées pour que quelque chose existe.

Ces conditions formelles ne sont justement pas matérielles. C'est ici que l'analogie avec les lois de la nature tombe. Le dispositif formel de l'ontologie (auquel appartiennent les concepts tels que "objet», "concept», "structure », " fait », " vérité », « réalité », " objectivité », " jugement », «inférence ", "sens », «identité », « existence», etc.) ne nous fournit aucun aperçu de ce qui meuble la réalité malgré le fait qu'il est lui-même quelque chose de réel. Il y a une différence entre une pierre et le fait qu'elle diffère de la neige qui la recouvre. Que la pierre soit couverte par la neige est un fait à propos de la pierre (et de la neige), et par conséquent une propriété de la pierre. Une des choses correctes que nous pouvons dire lorsque nous sommes en présence de cette situation est que la pierre est couverte de neige. Ni le fait que la pierre est couverte de neige, ni non plus le fait que nous disons la vérité lorsque nous le faisons remarquer, ne dépendent de l'esprit en un quelconque sens particulier.

Une fois que nous avons instauré un langage pour en parler, le réel peut être transformé en une série de normes. C’est alors le réel qui décide

18. Sur cette notion, voir Markus Gabriel, Limits of Epistemology, trad. A. Englander, Cambridge, Polity, 2018. 
lesquelles de nos expressions, en exprimant des pensées respectivement vraies ou fausses, sont en contact avec lui. Le réel est donc la norme de la réalité, et ce, dès que nous entrons en scène. D’après Frege, les lois de l'être vrai sont réelles et les sens frégéens le sont tout autant. Les actes humains de penser et de faire sens ne sont pas séparés de ce qui est. Grâce à notre adhésion à des ordres normatifs, nous n'appartenons pas à un domaine différent isolé de la nature par un écran. Nous, êtres pensants et connaissants, sommes simplement quelque chose de plus au sein de ce qu'il y a, différents des pierres, des arbres et des fermions, et même différents de notre propre cerveau. Mais c'est une autre histoire"19.

*Texte traduit par Marie-Hélène Desmeules.

19. Pour une plus ample discussion portant sur cette question et à laquelle Benoist et moi participons: Cf. Markus Gabriel, Neo-Existentialism, avec les commentaires de Jocelyn Maclure, Charles Taylor, Jocelyn Benoist, et Andrea Kern, suivi des réponses de l'auteur, Cambridge, Polity, 20I 8 . Une traduction française de cet ouvrage est actuellement en préparation et sera publiée aux Presses de l’Université Laval. 